Louisiana State University

LSU Digital Commons

7-1-2018

\title{
Bounded backstepping control and robustness analysis for time- varying systems under converging-input-converging-state conditions
}

\author{
Frédéric Mazenc \\ CentraleSupélec - Paris-Saclay \\ Michael Malisoff \\ Louisiana State University \\ Laurent Burlion \\ ONERA Centre de Toulouse \\ Jerome Weston \\ Louisiana State University
}

Follow this and additional works at: https://digitalcommons.Isu.edu/mathematics_pubs

\section{Recommended Citation}

Mazenc, F., Malisoff, M., Burlion, L., \& Weston, J. (2018). Bounded backstepping control and robustness analysis for time-varying systems under converging-input-converging-state conditions. European Journal of Control, 42, 15-24. https://doi.org/10.1016/j.ejcon.2018.02.005

This Article is brought to you for free and open access by the Department of Mathematics at LSU Digital Commons. It has been accepted for inclusion in Faculty Publications by an authorized administrator of LSU Digital Commons. For more information, please contact ir@lsu.edu. 
archives-ouvertes

\title{
Bounded Backstepping Control and Robustness Analysis for Time-Varying Systems under Converging- Input-Converging-State Conditions *
}

\author{
Frédéric Mazenc, Michael Malisoff, Laurent Burlion, Jerome Weston
}

\section{To cite this version:}

Frédéric Mazenc, Michael Malisoff, Laurent Burlion, Jerome Weston. Bounded Backstepping Control and Robustness Analysis for Time-Varying Systems under Converging- Input-Converging-State Conditions *. European Journal of Control, Elsevier, 2018, 42, pp.15-24. 10.1016/j.ejcon.2018.02.005. hal-01848927

\section{HAL Id: hal-01848927 \\ https://hal.inria.fr/hal-01848927}

Submitted on 25 Jul 2018

HAL is a multi-disciplinary open access archive for the deposit and dissemination of scientific research documents, whether they are published or not. The documents may come from teaching and research institutions in France or abroad, or from public or private research centers.
L'archive ouverte pluridisciplinaire HAL, est destinée au dépôt et à la diffusion de documents scientifiques de niveau recherche, publiés ou non, émanant des établissements d'enseignement et de recherche français ou étrangers, des laboratoires publics ou privés. 


\title{
Bounded Backstepping Control and Robustness Analysis for Time-Varying Systems under Converging- Input-Converging-State Conditions *
}

\author{
Frédéric Mazenc \\ Michael Malisoff \\ Laurent Burlion \\ Jerome Weston
}

August 17, 2017

\begin{abstract}
We provide new bounded backstepping results that ensure global asymptotic stability for a large class of partially linear systems with an arbitrarily large number of integrators. We use a dynamic extension that contains one artificial delay, and a converging-input-converging-state assumption. When the nonlinear subsystem is control affine, we provide sufficient conditions for our converging-input-converging-state assumption to hold. We also show input-to-state stability with respect to a large class of model uncertainties, and robustness to delays in the measurements of the state of the nonlinear subsystem. We illustrate our result using an example that is beyond the scope of classical backstepping.
\end{abstract}

\section{Introduction}

This paper continues our group's quest (begun in [12], [13], [14], [15], [18], and [19]) for novel backstepping results that help overcome the obstacles to using classical backstepping; see [8] and [10] for traditional backstepping. Classical backstepping entails synthesizing globally asymptotically stabilizing feedback controls, by recursively building globally asymptotically stabilizing controls and corresponding Lyapunov functions for subsystems; see [5], [7], [8], and [11] for improved backstepping theory that can include nonlinearities and uncertainties, and see [2], [3], and [4] for backstepping applied to adaptive, aerospace, and robotic systems. However, there are significant instances that call for backstepping but where the existing backstepping literature does not apply, e.g., systems with general nonlinear subsystems where there are bounds on the allowable sup norms of the controls, which produce challenges that we overcome in this work.

In this work, we focus on systems of the form

$$
\left\{\begin{aligned}
\dot{x}(t) & =\mathcal{F}(t, x(t), z(t), \eta(t)) \\
\dot{z}_{i}(t) & =z_{i+1}(t), \quad i \in\{1, \ldots, k-1\} \\
\dot{z}_{k}(t) & =u(t)+\sum_{j=1}^{k} v_{j} z_{j}(t)
\end{aligned}\right.
$$

with a scalar valued control $u$ and any number $k \geq 2$ of integrators, where $x$ is valued in $\mathbb{R}^{n}$ for any $n$, the $v_{j}$ 's are real constants, the unknown measurable essentially bounded function $\eta$ represents model uncertainty, and the nonlinear $x$ subsystem will satisfy a converging-input-converging-state condition that we specify below. Many nonlinear systems admit changes of variables that produce the form (1); see the well known results [6, Section 9.1] for formulas for the changes of coordinates, and Section 6 for examples that illustrate

\footnotetext{
*Key Words: Backstepping, delays, stabilization. Corresponding Author: Frédéric Mazenc, Tel.: + 33 [0] 169851762 , Fax: +33 [0] 169851765 . A preliminary version was accepted for presentation at the 2017 IEEE Conference on Decision and Control; see Section 1 for the differences between the conference version and this paper. Supported by NSF-ECCS Grants 1102348 and 1408295. Mazenc is with EPI DISCO INRIA-Saclay, L2S, CNRS CentraleSupelec, 3 rue Joliot Curie, 91192 Gifsur-Yvette, France (email: frederic.mazenc@12s.centralesupelec.fr). Malisoff and Weston are with Department of Mathematics, 303 Lockett Hall, Louisiana State University, Baton Rouge, LA 70803-4918, USA (email: malisoff@lsu.edu, jwesto3@lsu.edu). Burlion is with ONERA, The French Aerospace Lab, 31055 Toulouse, France (email: lburlion@onera.fr).
} 
the value of our theory. We write our controls as $u(t)$ to simplify notation, but our controls will be feedbacks that depend on $t$ through their dependence on states of the original system and of a dynamic extension.

In most of what follows, we assume that the current values of the state are available for measurement, but our main result will still use a delay in the state values in our feedback control since this so-called artificial delay is needed to design a bounded control; see Section 5 for an extension to cases where there are also delays in the measurements of the values $x(t)$ of the nonlinear subsystem of (1). Our work [15] also used both the converging-input-converging-state assumption and artificial delays, but a notable improvement in the present work as compared with [15] is that here we allow an arbitrarily large number $k$ of integrators, while [14, 15] only allowed one integrator. Although our bounded backstepping work [18] also allowed an arbitrarily large number of integrators, a notable advantage of the present work over [18] is that we produce a globally bounded control for (1) while the controls for the original systems in [18] were not globally bounded. Also, whereas [18] required $k$ artificial delays in the control and did not use dynamic extensions, here we only require one artificial delay, so in this sense we obtain a simpler feedback.

Our works [12], [13], [14] and [19] did not use converging-input-converging-state conditions or artificial delays. Moreover, our work differs from the backstepping works [13] (which uses a forwarding method to cover the one integrator case), [12] (which also only covers one integrator), [19] (which produces unbounded controls), and [22], [23], and [24] (which use Lie derivatives without satisfying the input constraints that we satisfy here). Therefore, our novel combination of converging-input-converging-state conditions with artificial delays and bounded controllers for (1) is valuable. The work to follow improves on our conference version [16] by also incorporating measurement delays and input-to-state stability with respect to the uncertainties $\eta$, and allowing the nonlinear subsystem to depend on all components of the vector $z$. These three features were not present in [16], which was confined to cases where $\mathcal{F}$ was a function of only $\left(t, x, z_{1}\right)$ and where there were no measurement delays and no input-to-state stability analysis.

We use standard notation and definitions. We omit arguments of functions when they are clear, and the dimensions of our Euclidean spaces are arbitrary unless otherwise noted. We use $|\cdot|$ to denote the usual Euclidean norm and the induced matrix norm, and $|\phi|_{\infty}$ (resp., $|\phi|_{\mathcal{I}}$ ) is the essential supremum (resp., supremum over any interval $\mathcal{I}$ ) for any bounded measurable function $\phi$. We set $\mathbb{N}=\{1,2, \ldots\}$. Given any constant $T>0$, let $C_{\text {in }}$ denote the set of all continuous functions $\phi:[-T, 0] \rightarrow \mathbb{R}^{a}$, which we call the set of all initial functions. We define $\Xi_{t} \in C_{\text {in }}$ and $\dot{\Xi}_{t} \in C_{\text {in }}$ by $\Xi_{t}(s)=\Xi(t+s)$ and $\dot{\Xi}_{t}(s)=\Xi^{\prime}(t+s)$ for all choices of $\Xi, s \leq 0$, and $t \geq 0$ for which the equalities are defined. We use the convention $0 !=1$, and assume for simplicity that the initial times for our solutions are $t_{0}=0$ and that the initial functions are constant at time 0 (e.g., the states are constant on $[-T, 0]$, where $T$ will denote the artificial delay). We let $f^{(i)}$ denote the $i$ th derivative of a function $f:[0,+\infty) \rightarrow \mathbb{R}$ with $f^{(0)}=f$, and $\sigma_{r}: \mathbb{R} \rightarrow[-r, r]$ is the saturation that is defined for all constants $r>0$ by $\sigma_{r}(s)=s$ for all $s \in[-r, r]$ and $\sigma_{r}(s)=r \operatorname{sign}(s)$ otherwise. An integral $\int_{a} J(\ell) \mathrm{d} \ell$ of a continuous column vector valued function $J=\left(J_{1}, \ldots J_{L}\right)^{\top}$ on an interval $a$ is defined to be the column vector whose $i$ th entry is $\int_{a} J_{i}(\ell) \mathrm{d} \ell$ for all $i$. We use the standard definitions of global asymptotic and input-to-state stability (or ISS, which we also use to mean input-to-state stable) [8].

\section{Lemmas and Main Result}

We require the following two lemmas, the first of which follows from elementary calculations that we omit:

Lemma 1. Let $q>0$ and $T>0$ be constants, and $\mu_{0}:[-T,+\infty) \rightarrow \mathbb{R}$ be any continuous function, and set

$$
\begin{aligned}
& \zeta(t)=\int_{t-T}^{t} e^{q(\ell-t)} Q(t, \ell, \ell+T) \mu_{0}(\ell) \mathrm{d} \ell \\
& \Omega_{j}(t)=\zeta^{(j-1)}(t) \text { and } \mu_{i}(t)=\frac{1}{T} \int_{t-T}^{t} e^{q(\ell-t)} \frac{(t-\ell)^{i-1}}{(i-1) !} \mu_{0}(\ell) \mathrm{d} \ell
\end{aligned}
$$

for all $j \in\{1, \ldots, k+1\}$ and $i \in \mathbb{N}$, where $Q(t, a, b)=(t-a)^{k-1}(t-b)^{k-1}$ for all $a \in \mathbb{R}$ and $b \in \mathbb{R}$ and $k \in \mathbb{N}$ with $k \geq 2$. Then there are constants $c_{i, j}(q, T) \in \mathbb{R}$ for all $i \in\{1, \ldots, 2 k-1\}$ and all $j \in\{1, \ldots, k\}$, and constants $g_{i}(q, T) \in \mathbb{R}$ for all $i \in\{-1,0, \ldots, 2 k-1\}$, such that

$$
\Omega_{j}(t)=\sum_{i=1}^{2 k-1} c_{i, j}(q, T) \mu_{i}(t) \text { and } \Omega_{k+1}(t)=\sum_{i=0}^{2 k-1} g_{i}(q, T) \mu_{i}(t)+g_{-1}(q, T) \mu_{0}(t-T)
$$

hold for all $t \geq 0$. 
Lemma 1 follows by first using the Binomial Theorem to write $Q(t, \ell, \ell+T)$ as a linear combination of the terms $(t-\ell)^{r}$ with $k-1 \leq r \leq 2 k-2$. In the next lemma (which was shown in [21]), we say that a linear system is not exponentially unstable provided its poles are all in the closed left-half plane:

Lemma 2. Let $k \geq 2$ be an integer and $v=\left(v_{1}, \ldots, v_{k}\right)$ be any vector of $k$ real constants such that

$$
\left\{\begin{aligned}
\dot{z}_{i}(t) & =z_{i+1}(t), \quad i \in\{1, \ldots, k-1\} \\
\dot{z}_{k}(t) & =u+\sum_{i=1}^{k} v_{i} z_{i}
\end{aligned}\right.
$$

is not exponentially unstable when $u=0$. Then there is a bounded locally Lipschitz function $\vartheta: \mathbb{R}^{k} \rightarrow \mathbb{R}$ such that (4) in closed loop with $u=\vartheta(Z)$ where $Z=\left(z_{1}, \ldots, z_{k}\right)^{\top}$ is globally asymptotically and locally exponentially stable to 0.

We can now fix functions $\Lambda_{j}$ such that the $\Omega_{j}$ 's from Lemma 1 can be written as

$$
\Omega_{j}(t)=\int_{t-T}^{t} \Lambda_{j}(\ell, t) \mu_{0}(\ell) \mathrm{d} \ell \text { for } 1 \leq j \leq k \text { and all } t \geq 0
$$

for all choices of the continuous function $\mu_{0}:[-T,+\infty) \rightarrow \mathbb{R}$, where we omit the dependence of the $\Lambda_{j}$ 's on $q$ and $T$ for brevity. For instance, we have

$$
\begin{aligned}
\Lambda_{1}(\ell, t)= & e^{q(\ell-t)}(t-\ell)^{k-1}(t-\ell-T)^{k-1} \text { and } \\
\Lambda_{2}(\ell, t)= & e^{q(\ell-t)}\left[-q(t-\ell)^{k-1}(t-\ell-T)^{k-1}+(k-1)(t-\ell)^{k-2}(t-\ell-T)^{k-1}\right. \\
& \left.+(k-1)(t-\ell)^{k-1}(t-\ell-T)^{k-2}\right]
\end{aligned}
$$

and the formulas for the other $\Lambda_{j}$ 's can be computed from Lemma 1. Notice for later use that the $\Lambda_{i}$ 's are all bounded on $[t-T, t]$ for each $t \geq 0$ and $T>0$. For instance, when $k=2$, we have

$$
\max \left\{\left|\Lambda_{j}(\ell, t)\right|: 1 \leq j \leq 2, \ell \in[t-T, t], t \geq 0\right\} \leq \max \left\{T^{2}, T(q T+2)\right\}
$$

for all $q>0$. Also, the $\Lambda_{i}$ 's can be rewritten as functions of $t-\ell$ and $t-\ell-T$. We will assume the following, where $\Lambda=\left(\Lambda_{1}, \ldots, \Lambda_{k}\right)^{\top}$ :

Assumption 1. (i) The function $\mathcal{F}$ in (1) is continuous in $t$ and $\eta$, globally Lipschitz in $(x, z)$, and satisfies

$$
\mathcal{F}(t, 0,0,0)=0 \text { for all } t \geq 0 .
$$

(ii) There is a globally Lipschitz bounded function $\omega: \mathbb{R}^{n} \rightarrow[-\bar{\omega}, \bar{\omega}]$ having some bound $\bar{\omega}>0$ such that $\omega(0)=0$ and constants $T>0$ and $q>0$ such that for each continuous function $\delta:[0,+\infty) \rightarrow \mathbb{R}^{k}$ that exponentially converges to 0 , the following is true: All solutions $\xi:[0,+\infty) \rightarrow \mathbb{R}^{n}$ of the system

$$
\dot{\xi}(t)=\mathcal{F}\left(t, \xi(t), \int_{t-T}^{t} \Lambda(\ell, t) \omega(\xi(\ell)) \mathrm{d} \ell+\delta(t), 0\right)
$$

satisfy $\lim _{t \rightarrow+\infty} \xi(t)=0$.

We refer to part (ii) of Assumption 1 as our converging-input-converging-state assumption; see Section 3 for sufficient conditions for Assumption 1 to hold, and see Section 5 for a generalization involving measurement delays in the $\xi$ measurements in the function $\omega$. The system (9) differs from the nonlinear subsystem of (1) because the third argument of $\mathcal{F}$ in (1) has been replaced by the sum of an integral term and $\delta(t)$, and because $\eta$ has been set to 0 . In terms of the Jordan matrix

$$
J_{2 k-1}=\left[\begin{array}{ccccc}
-q & 1 & 0 & \ldots & 0 \\
0 & -q & 1 & \ldots & 0 \\
\vdots & \ddots & \ddots & \ddots & \vdots \\
\vdots & & \ddots & -q & 1 \\
0 & \ldots & \ldots & 0 & -q
\end{array}\right] \in \mathbb{R}^{(2 k-1) \times(2 k-1)}
$$

our main result is as follows, where the forward completeness of the closed loop system follows from the boundedness of $\omega$ and of the control, and the global Lipschitzness properties of $\mathcal{F}$ and $\omega$ : 
Theorem 1. Let the constants $q>0, k \geq 2$, and $T>0$ and the functions $\mathcal{F}$ and $\omega$ be such that Assumption 1 holds, where $k \in \mathbb{N}$. Let $\vartheta$ and $v$ satisfy the requirements from Lemma 2. Consider the augmented (x, Z, Y) system, consisting of (1) and

$$
\dot{Y}(t)=J_{2 k-1} Y(t)+\frac{e_{2 k-1}}{T} \omega(x(t))
$$

where $e_{2 k-1}=(0,0, \ldots, 1)^{\top} \in \mathbb{R}^{2 k-1}$ is the $(2 k-1)$-st standard basis vector, in closed loop with the control

$$
u\left(Z(t), Y_{t}, x_{t}\right)=\sigma_{\bar{c}}\left(\mathcal{M}\left(Y_{t}\right)\right)+g_{0}(q, T) \omega(x(t))+g_{-1}(q, T) \omega(x(t-T))+\vartheta\left(Z_{\star}(t)\right)
$$

with the saturation level

$$
\bar{c}=\left|\sum_{j=1}^{k} v_{j} \mathcal{C}_{j}(q, T)-\mathcal{G}(q, T)\right| e^{\left|J_{2 k-1}\right| T \bar{\omega}}
$$

where $Z_{\star}(t)=\left(z_{1}(t)-\mathcal{C}_{1}(q, T) \Psi\left(Y_{t}\right), \ldots, z_{k}(t)-\mathcal{C}_{k}(q, T) \Psi\left(Y_{t}\right)\right)^{\top}, \Psi\left(Y_{t}\right)=Y(t)-e^{T J_{2 k-1}} Y(t-T), \mathcal{G}(q, T)=$ $\left[g_{2 k-1}(q, T) \ldots \ldots g_{1}(q, T)\right]$, and

$$
\mathcal{M}\left(Y_{t}\right)=\left(\mathcal{G}(q, T)-\sum_{j=1}^{k} v_{j} \mathcal{C}_{j}(q, T)\right) \Psi\left(Y_{t}\right) \text { and } \mathcal{C}_{j}(q, T)=\left[c_{2 k-1, j}(q, T) \ldots \ldots c_{1, j}(q, T)\right], 1 \leq j \leq k
$$

and where the constants $c_{i, j}$ and $g_{i}$ satisfy the requirements from Lemma 1 for the function $\mu_{0}(t)=\omega(x(t))$. Then all maximal solutions $(x, Z, Y)(t)$ of the augmented $(x, Z, Y)$ system satisfy $\lim _{t \rightarrow+\infty}(x, Z, Y)(t)=0$ when $\eta=0$. If, in addition, the system

$$
\dot{\xi}(t)=\mathcal{F}\left(t, \xi(t), \int_{t-T}^{t} \Lambda(\ell, t) \omega(\xi(\ell)) \mathrm{d} \ell+\delta(t), \eta(t)\right)
$$

is ISS with respect to $(\delta, \eta)$, then the closed loop $(x, z)$ system is ISS with respect to $\eta$.

Remark 1. As in [18], we can extend Theorem 1 to cases where in addition to the artificial delay $T$, there is a delay in the measurements of $x(t)$ from the original system (1). However, as we noted above, [18] does not provide a bounded control for (1) even if the $v_{i}$ 's are all zero, and the converging-input-converging-state assumption in [18] has a $k$-fold integral instead of the simpler single integral we have in (9).

We next provide sufficient conditions for our converging-input-converging-state assumption to hold, and then we prove Theorem 1 in Section 4. See also Section 5 for extensions under measurement delays.

\section{Checking Assumption 1}

We provide sufficient conditions for our converging-input-converging-state conditions on (9) to hold, and for the ISS property of (15) from Theorem 1 to hold, based on Lyapunov functions. We use the system

$$
\dot{x}(t)=\mathcal{F}\left(t, x(t), \Lambda_{*}(T) \omega(x(t)), \eta(t)\right),
$$

where $\mathcal{F}$ is from $(1), \Lambda_{*}:[0, \infty) \rightarrow \mathbb{R}^{p}$ is defined by

$$
\Lambda_{*}(T)=\int_{t-T}^{t}\left(\Lambda_{1}(\ell, t), \ldots, \Lambda_{p}(\ell, t)\right)^{\top} \mathrm{d} \ell,
$$

the constant $T>0$ will be specified, the functions $\Lambda_{i}$ 's satisfy the requirements from (5), and $p \in[1, k]$ is such that $\mathcal{F}$ is a function of $\left(t, x, z_{1}, \ldots, z_{p}, \eta\right)$, where $z_{1}, \ldots, z_{p}$ are the first $p$ components of the state $z$ of the linear subsystem of (1). The definition (17) is justified by the fact that each function $\Lambda_{i}(\ell, t)$ for $i=1,2, \ldots, p$ can be written as a function of $t-\ell-T$ and $t-\ell$, so the right side of (17) does not depend on $t$. We also use the following standard definitions. A function $W: \mathbb{R}^{n} \rightarrow[0,+\infty)$ is called positive definite provided $W(0)=0$ and $W(x)>0$ for all $x \in \mathbb{R}^{n} \backslash\{0\}$. A function $V:[0,+\infty) \times \mathbb{R}^{n} \rightarrow[0,+\infty)$ is called uniformly proper and positive definite provided there are functions $\alpha_{0} \in \mathcal{K}_{\infty}$ and $\alpha_{1} \in \mathcal{K}_{\infty}$ such that the inequalities $\alpha_{0}(|x|) \leq V(t, x) \leq \alpha_{1}(|x|)$ hold for all $t \geq 0$ and $x \in \mathbb{R}^{n}$. This agrees with the properness condition in the special case where $V$ is independent of $t$. Here, $\mathcal{K}_{\infty}$ is the set of all continuous functions $\gamma:[0,+\infty) \rightarrow[0,+\infty)$ such that $\gamma(0)=0, \gamma$ is strictly increasing, and $\lim _{s \rightarrow+\infty} \gamma(s)=+\infty$. In the next assumption, $V_{t}$ and $V_{x}$ are the partial derivative with respect to $t$ and the gradient with respect to $x$, respectively, and the uniform global Lipschitzness in $x$ means that the global Lipschitz constants can be chosen independently of the other variable $t$ : 
Assumption 2. There are functions $f:[0,+\infty) \times \mathbb{R}^{n} \rightarrow \mathbb{R}^{n}$ and $g:[0,+\infty) \times \mathbb{R}^{n} \rightarrow \mathbb{R}^{n \times p}$ that are uniformly globally Lipschitz in $x$ and continuous on $[0,+\infty) \times \mathbb{R}^{n}$, such that $\mathcal{F}(t, x, q, \eta)=f(t, x)+g(t, x)(q+\eta)$ holds for all $t \geq 0, x \in \mathbb{R}^{n}, q \in \mathbb{R}^{p}$, and $\eta \in \mathbb{R}^{p}$. There exist a $C^{1}$ uniformly proper and positive definite function $V:[0,+\infty) \times \mathbb{R}^{n} \rightarrow[0,+\infty)$; a uniformly continuous positive definite function $W: \mathbb{R}^{n} \rightarrow[0,+\infty)$; positive constants $T, r_{0}, r_{1}$, and $r_{3}$; and a constant $r_{2} \geq 0$ such that for all $(t, x) \in[0,+\infty) \times \mathbb{R}^{n}$, we have

$$
\begin{aligned}
& V_{t}(t, x)+V_{x}(t, x)\left(f(t, x)+g(t, x) \Lambda_{*}(T) \omega(x)\right) \leq-W(x), \\
& \left|V_{x}(t, x) g(t, x)\right| \leq r_{0} \sqrt{W(x)},|\omega(x)| \leq r_{1} \sqrt{W(x)},|f(t, x)| \leq r_{2} \sqrt{W(x)}, \text { and }|g(t, x)| \leq r_{3},
\end{aligned}
$$

where $\omega: \mathbb{R}^{n} \rightarrow \mathbb{R}$ is bounded, satisfies $\omega(0)=0$, and admits a global Lipschitz constant $C>0$ on $\mathbb{R}^{n}$.

See [17] for conditions under which (18) can be satisfied. Set

$$
\Lambda_{a}(T)=\int_{t-T}^{t}\left|\left(\Lambda_{1}, \ldots, \Lambda_{p}\right)(\ell, t)\right| \mathrm{d} \ell,
$$

which is independent of $t$ because the $\Lambda_{i}$ 's can be written as functions of $t-\ell$ and $t-\ell-T$. We also set $\Lambda_{+}(T)=\sup _{t \geq 0}\left\{\left|\left(\Lambda_{1}(\ell, t), \ldots, \Lambda_{p}(\ell, t)\right)\right|: t-T \leq \ell \leq t\right\}$, which is finite because of our choice of $\Lambda$.

Proposition 1. If Assumption 2 holds, then for all integers $k \geq 2$, and for all constants $T>0$ such that

$$
4\left(T \Lambda_{a}(T) r_{0} C\right)^{2}\left[2 r_{2}^{2}+\frac{5}{2}\left(r_{1} r_{3} T \Lambda_{+}(T)\right)^{2}\right]<1,
$$

Assumption 1 is satisfied. If, in addition, $W$ is proper, then (15) is ISS with respect to $(\delta, \eta)$.

Proof. We first prove the first assertion of the proposition (where $\eta=0$ ), and then we indicate the additional arguments needed to prove the second assertion. Fix any continuous function $\delta:[0,+\infty) \rightarrow \mathbb{R}^{p}$ that exponentially converges to 0 . Along all solutions $x(t)$ of $(9)$, the control affine structure of $\mathcal{F}$ gives

$$
\dot{x}(t)=f(t, x(t))+g(t, x(t)) \Lambda_{*} \omega(x(t))+g(t, x(t))\left[\delta(t)+\int_{t-T}^{t} \Lambda^{b}(\ell, t)(\omega(x(\ell))-\omega(x(t))) \mathrm{d} \ell\right]
$$

where $\Lambda^{b}=\left(\Lambda_{1}, \ldots, \Lambda_{p}\right)^{\top}$, and therefore also

$$
\dot{V}(t) \leq-W(x(t))+\sqrt{W(x(t))}\left(\left\{\Lambda_{a} r_{0} \sup _{\ell \in[t-T, t]}|\omega(x(\ell))-\omega(x(t))|\right\}+r_{0}|\delta(t)|\right),
$$

where we will omit the argument $T$ of $\Lambda_{a}$ and $\Lambda_{+}$for brevity. We next use the global Lipschitz constant $C$ on $\omega$ and apply the Fundamental Theorem of Calculus to find a useful upper bound on the supremum from (22). To this end, we first use Assumption 2 to obtain the upper bound

$$
|\dot{x}(t)| \leq r_{2} \sqrt{W(x(t))}+r_{3}\left(\left|\Lambda_{+}\right| \int_{t-T}^{t}|\omega(x(\ell))| \mathrm{d} \ell+|\delta(t)|\right)
$$

along all solutions of (9). Applying $(a+b)^{2} \leq 2\left(a^{2}+b^{2}\right)$ and then $(a+b)^{2} \leq(5 / 4) a^{2}+5 b^{2}$ for suitable $a \geq 0$ and $b \geq 0$ and then Jensen's inequality and Assumption 2 that along all solutions of (21), we have

$$
\begin{aligned}
|\dot{x}(t)|^{2} & \leq 2 r_{2}^{2} W(x(t))+2 r_{3}^{2}\left(5|\delta(t)|^{2}+\frac{5}{4}\left(\int_{t-T}^{t} \Lambda^{b}(\ell, t) \omega(x(\ell)) \mathrm{d} \ell\right)^{2}\right) \\
& \leq 2 r_{2}^{2} W(x(t))+2 r_{3}^{2}\left(5|\delta(t)|^{2}+\frac{5}{4} \operatorname{Tr}_{1}^{2} \Lambda_{+}^{2} \int_{t-T}^{t} W(x(\ell)) \mathrm{d} \ell\right) .
\end{aligned}
$$

We can now combine $(22)$ and (24) and then use Jensen's and Young's inequalities $\sqrt{W(x(t))} b \leq$ $\frac{1}{4} W(x(t))+b^{2}$, with $b=r_{0}|\delta(t)|$ and then $b$ being the quantity in curly braces in (22), to get

$$
\begin{aligned}
\dot{V}(t) \leq & -\frac{1}{2} W(x(t))+r_{0}^{2}\left(|\delta(t)|^{2}+\Lambda_{a}^{2} C^{2} \sup _{\ell \in[t-T, t]}|x(\ell)-x(t)|^{2}\right) \\
\leq & -\frac{1}{2} W(x(t))+r_{0}^{2}\left(|\delta(t)|^{2}+\Lambda_{a}^{2} C^{2} T \int_{t-T}^{t}|\dot{x}(\ell)|^{2}\right) \\
\leq & -\frac{1}{2} W(x(t))+r_{0}^{2}\left(|\delta(t)|^{2}+\Lambda_{a}^{2} C^{2} T\left[2 r_{2}^{2} \int_{t-T}^{t} W(x(\ell)) \mathrm{d} \ell+10 r_{3}^{2} T|\delta|_{[t-T, t]}^{2}\right.\right. \\
& \left.\left.+\frac{5}{2} r_{3}^{2} T^{2} \Lambda_{+}^{2} r_{1}^{2} \int_{t-2 T}^{t} W(x(\ell)) \mathrm{d} \ell\right]\right) \\
\leq & -\frac{1}{2} W(x(t))+\mathcal{N}_{1} \int_{t-2 T}^{t} W(x(\ell)) \mathrm{d} \ell+\mathcal{N}_{2}|\delta|_{[t-T, t]}^{2}
\end{aligned}
$$


along all solutions of (9) for all $t \geq 0$, where

$$
\mathcal{N}_{1}=T\left(r_{0} \Lambda_{a} C\right)^{2}\left(2 r_{2}^{2}+\frac{5}{2}\left(r_{1} r_{3} \Lambda_{+} T\right)^{2}\right)
$$

and $\mathcal{N}_{2}=10\left(r_{0} T \Lambda_{a} r_{3} C\right)^{2}+r_{0}^{2}$, by using the bound $\int_{s-T}^{s} W(x(\ell)) \mathrm{d} \ell \leq \int_{t-2 T}^{t} W(x(\ell)) \mathrm{d} \ell$ for all $s \in[t-T, t]$. Then our condition (20) implies that $2 T \mathcal{N}_{1}<1 / 2$, so we can find a constant $\lambda>1$ that is close enough to 1 so that $2 T \mathcal{N}_{1} \lambda<1 / 2$. Then the function

$$
V_{1}\left(t, x_{t}\right)=V(t, x(t))+\lambda \mathcal{N}_{1} \int_{t-2 T}^{t} \int_{s}^{t} W(x(\ell)) \mathrm{d} \ell \mathrm{d} s
$$

satisfies

$$
\dot{V}_{1} \leq-\left\{\frac{1}{2}-2 T \mathcal{N}_{1} \lambda\right\} W(x(t))-(\lambda-1) \mathcal{N}_{1} \int_{t-2 T}^{t} W(x(\ell)) \mathrm{d} \ell+\mathcal{N}_{2}|\delta|_{[t-T, t]}^{2}
$$

for all $t \geq 0$ along all solutions of (9), since

$$
\frac{d}{d t} \int_{t-2 T}^{t} \int_{s}^{t} W(x(\ell)) \mathrm{d} \ell \mathrm{d} s=2 T W(x(t))-\int_{t-2 T}^{t} W(x(\ell)) \mathrm{d} \ell,
$$

holds for all $t \geq 0$.

Since the quantity in curly braces in (28) is positive and $\lambda>1$, and since our assumptions on $\delta$ ensure that $|\delta|_{[t-T, t]}^{2}$ is integrable, we can integrate $(28)$ on $[0, M]$ for any $M>0$ to get $\sup _{t>0} V_{1}\left(t, x_{t}\right)<+\infty$. Since $V$ is uniformly proper and positive definite, we conclude that $|x(t)|$ is bounded, so $x(t)$ is uniformly continuous, by the structure of the dynamics (9) when $\eta=0$. Since $W$ is uniformly continuous, it follows that $W(x(t))$ is a uniformly continuous function of $t$, and integrating (28) gives

$$
\int_{0}^{+\infty} W(x(\ell)) \mathrm{d} \ell<+\infty .
$$

Therefore, Barbalat's Lemma implies that $\lim _{t \rightarrow+\infty} W(x(t))=0$, so since $W$ is positive definite, we conclude that $\lim _{t \rightarrow+\infty} x(t)=0$. This proves the first assertion of the proposition.

To prove the second assertion of the proposition, fix a choice of the measurable essentially bounded function $\eta$. Then the preceding analysis applies to the corresponding system (15), save for the fact that we must add the additional term $V_{x}(t, x(t)) g(t, x(t)) \eta(t)$ to the right sides of the decay estimates on $V$. We can use Jensen's inequality to check that this additional term is bounded above by

$$
r_{0} \sqrt{W(x(t))}|\eta(t)| \leq \frac{c_{*}}{2} W(x(t))+\frac{1}{2 c_{*}} r_{0}^{2}|\eta(t)|^{2}
$$

where $c_{*}>0$ is the constant in curly braces in (28). If we add the right side of (31) to the right side of (28) and use the fact that

$$
\int_{t-2 T}^{t} \int_{s}^{t} W(x(\ell)) \mathrm{d} \ell \mathrm{d} s \leq 2 T \int_{t-2 T}^{t} W(x(\ell)) \mathrm{d} \ell
$$

for all $t \geq 0$, then we can find a function $\gamma_{0} \in \mathcal{K}_{\infty}$ and a constant $k_{*}>0$ such that

$$
\dot{V}_{1} \leq-\gamma_{0}\left(V_{1}\left(t, x_{t}\right)\right)+k_{*}|(\delta, \eta)|_{[0, t]}^{2}
$$

along all solutions of (9), using the properness of $V$ and $W$ to find a $\gamma_{1} \in \mathcal{K}_{\infty}$ such that $\gamma_{1}(V(t, x)) \leq$ $\left(c_{*} / 2\right) W(x)$ for all $t \geq 0$ and $x \in \mathbb{R}^{n}$, then choosing $\gamma_{0}(\ell)=\min \left\{\gamma_{1}(\ell / 2), r_{*} \ell\right\}$ with $r_{*}=(\lambda-1) /(4 T \lambda)$ (by the relation $\gamma_{0}(a+b) \leq \gamma_{0}(2 a)+\gamma_{0}(2 b)$ where $a$ and $b$ are the terms being added in the formula (27)). Hence, $V_{1}$ is an ISS Lyapunov function for the system, so the ISS properties follow by standard arguments [8].

Remark 2. Proposition 2 requires $T>0$ to be small enough, but due to the structure of our controller in Theorem 1, we cannot pick $T=0$. In Section 5, we will see how picking $T$ small enough can ensure that the ISS property is maintained even when there are measurement delays $D$ in the $x$ values in our feedbacks.

\section{Proof of Theorem 1}

Theorem 1 will follow from three more lemmas, which we state next. The first of these lemmas follows from [20, Lemma A.3.2] (applied to the entire function $\mathcal{E}(x)=e^{x t}$ for any $t \in \mathbb{R}$ to compute $\mathcal{E}\left(J_{2 k-1}\right)$ ): 
Lemma 3. For the Jordan matrix $J_{2 k-1}$ defined in (10), the equality

$$
e^{J_{2 k-1} t}=e^{-q t}\left[\begin{array}{ccccc}
1 & t & \frac{t^{2}}{2} & \ldots & \frac{t^{2(k-1)}}{(2(k-1)) !} \\
0 & 1 & t & \ldots & \frac{t^{2 k-3}}{(2 k-3) !} \\
\vdots & \ddots & \ddots & \ddots & \vdots \\
\vdots & & \ddots & 1 & t \\
0 & \ldots & \ldots & 0 & 1
\end{array}\right]
$$

holds for all $t \in \mathbb{R}$ and integers $k \geq 2$.

Later in the proof of Theorem 1, we specialize the following lemma to the case where $\mu_{0}(t)=\omega(x(t))$ :

Lemma 4. Let $\mu_{0}:[-T,+\infty) \rightarrow[-\bar{\mu}, \bar{\mu}]$ be any continuous function having a bound $\bar{\mu}$. Then the functions $\mu_{i}$ from (2) in Lemma 1, and the functions $\Psi\left(Y_{t}\right)=Y(t)-e^{T J_{2 k-1}} Y(t-T)$ for all solutions $Y$ of

$$
\dot{Y}(t)=J_{2 k-1} Y(t)+\frac{e_{2 k-1}}{T} \mu_{0}(t)
$$

are such that for all $t \geq 0$, we have

$$
\nu_{2 k-1}(t)=\Psi\left(Y_{t}\right) \text { and }\left|\Psi\left(Y_{t}\right)\right| \leq e^{\left|J_{2 k-1}\right| T} \bar{\mu},
$$

where $\nu_{2 k-1}(t)=\left(\mu_{2 k-1}(t), \ldots, \mu_{1}(t)\right)^{\top}$ for all $t \geq 0$.

Proof. By integrating (35), we deduce that

$$
\Psi\left(Y_{t}\right)=Y(t)-e^{T J_{2 k-1}} Y(t-T)=\varrho(t), \text { where } \varrho(t)=\int_{t-T}^{t} e^{J_{2 k-1}(t-\ell) \frac{e_{2 k-1}}{T} \mu_{0}(\ell) \mathrm{d} \ell}
$$

for all $t \geq 0$. On the other hand, using (34), we obtain

$$
\varrho(t)=\frac{1}{T} \int_{t-T}^{t} e^{-q(t-\ell)}\left[\begin{array}{c}
\frac{(t-\ell)^{2(k-1)}}{(2(k-1)) !} \\
\vdots \\
t-\ell \\
1
\end{array}\right] \mu_{0}(\ell) d \ell=\nu_{2 k-1}(t),
$$

which proves the first conclusion of the lemma. The second conclusion of the lemma follows since (37) gives the estimate

$$
\left|Y(t)-e^{T J_{2 k-1}} Y(t-T)\right| \leq \int_{t-T}^{t}\left|e^{J_{2 k-1}(t-\ell)} \frac{e_{2 k-1}}{T}\right| \bar{\mu} \mathrm{d} \ell
$$

for all $t \geq 0$, which proves the lemma.

Lemma 5. Let $\mu_{0}:[-T,+\infty) \rightarrow[-\bar{\mu}, \bar{\mu}]$ be any continuous function having a bound $\bar{\mu}$, and let the constants $v_{i}$ and the function $\vartheta$ satisfy the requirements from Lemma 2. Consider the linear system

$$
\left\{\begin{array}{l}
\dot{z}_{i}(t)=z_{i+1}(t), \quad i \in\{1, \ldots, k-1\} \\
\dot{z}_{k}(t)=u(t)+\sum_{j=1}^{k} v_{j} z_{j}(t)
\end{array}\right.
$$

in closed loop with the control

$$
u\left(Z(t), Y_{t}, x_{t}\right)=\sigma_{\bar{c}}\left(\mathcal{M}\left(Y_{t}\right)\right)+g_{0}(q, T) \mu_{0}(t)+g_{-1}(q, T) \mu_{0}(t-T)+\vartheta\left(Z_{\star}(t)\right)
$$

with the saturation level $\bar{c}$ for $\sigma_{\bar{c}}$ defined by

$$
\bar{c}=\left|\sum_{j=1}^{k} v_{j} \mathcal{C}_{j}(q, T)-\mathcal{G}(q, T)\right| e^{\left|J_{2 k-1}\right| T} \bar{\mu}
$$

and where $Y$ satisfies (35) and $\mathcal{M}, Z_{\star}, \mathcal{G}$, and the $\mathcal{C}_{j}$ 's and $g_{j}$ 's are defined as in Theorem 1. Then the dynamics for the vector $\tilde{Z}(t)=\left(\tilde{z}_{1}(t), \ldots, \tilde{z}_{k}(t)\right)$ are globally asymptotically and locally exponentially stable to the origin, where $\tilde{z}_{i}(t)=z_{i}(t)-\Omega_{i}(t)$ for $i=1,2, \ldots, k$ and the $\Omega_{i}$ 's are defined in (2) in Lemma 1. 
Proof. The fact that $\dot{\Omega}_{i}=\Omega_{i+1}$ for all $i \in\{1,2, \ldots, k\}$ and the structure of the dynamics (40) allow us to conclude that the dynamics for the functions $\tilde{z}_{i}(t)=z_{i}(t)-\Omega_{i}(t)$ are

$$
\left\{\begin{array}{l}
\dot{\tilde{z}}_{i}(t)=\tilde{z}_{i+1}(t), \quad i \in\{1, \ldots, k-1\} \\
\dot{\tilde{z}}_{k}(t)=u(t)-\Omega_{k+1}(t)+\sum_{j=1}^{k} v_{j}\left[\tilde{z}_{j}(t)+\Omega_{j}(t)\right] .
\end{array}\right.
$$

Using our conclusion from Lemma 4 that

$$
\nu_{2 k-1}(t)=\Psi\left(Y_{t}\right)
$$

where $\nu_{2 k-1}(t)=\left(\mu_{2 k-1}(t), \ldots, \mu_{1}(t)\right)^{\top}$ as before, it follows from (3) that

$$
\begin{aligned}
\dot{\tilde{z}}_{k}(t)= & u(t)-\mathcal{G}(q, T) \nu_{2 k-1}(t)-g_{0}(q, T) \mu_{0}(t)-g_{-1}(q, T) \mu_{0}(t-T) \\
& +\sum_{j=1}^{k} v_{j} \tilde{z}_{j}(t)+\sum_{j=1}^{k} v_{j} \mathcal{C}_{j}(q, T) \nu_{2 k-1} .
\end{aligned}
$$

Hence, (44) gives

$$
\left\{\begin{array}{l}
\dot{\tilde{z}}_{i}(t)=\tilde{z}_{i+1}(t), \quad i \in\{1, \ldots, k-1\} \\
\dot{\tilde{z}}_{k}(t)=u(t)+\sum_{j=1}^{k} v_{j} \tilde{z}_{j}(t)-g_{0}(q, T) \mu_{0}(t)-g_{-1}(q, T) \mu_{0}(t-T)+\bar{g} \Psi\left(Y_{t}\right)
\end{array}\right.
$$

where

$$
\bar{g}=\sum_{j=1}^{k} v_{j} \mathcal{C}_{j}(q, T)-\mathcal{G}(q, T) .
$$

Next note that since Lemma 1 gives $\Omega_{j}=\mathcal{C}_{j}(q, T) \nu_{2 k-1}$ for $j=1, \ldots, k$, it follows that

$$
\tilde{z}_{i}(t)=z_{i}(t)-\Omega_{i}(t)=z_{i}(t)-\mathcal{C}_{i}(q, T) \nu_{2 k-1}(t) \text { for } i \in\{1, \ldots, k\} .
$$

Thus, (44) gives $\tilde{z}_{i}(t)=z_{i}(t)-\mathcal{C}_{i}(q, T) \Psi\left(Y_{t}\right)$ for all $t \geq 0$ and all $i \in\{1, \ldots, k\}$, so $Z_{\star}(t)=\tilde{Z}(t)$ for all $t \geq 0$. Also, $\mathcal{M}\left(Y_{t}\right)=-\bar{g} \Psi\left(Y_{t}\right)$. Therefore, our choice (41) of the control gives

$$
\left\{\begin{array}{l}
\dot{\tilde{z}}_{i}(t)=\tilde{z}_{i+1}(t), \quad i \in\{1, \ldots, k-1\} \\
\dot{\tilde{z}}_{k}(t)=\sum_{j=1}^{k} v_{j} \tilde{z}_{j}(t)+\sigma_{\bar{c}}\left(-\bar{g} \Psi\left(Y_{t}\right)\right)+\bar{g} \Psi\left(Y_{t}\right)+\vartheta\left(Z_{\star}(t)\right) .
\end{array}\right.
$$

According to (36), we have

$$
\left|\bar{g} \Psi\left(Y_{t}\right)\right| \leq|\bar{g}| e^{\left|J_{2 k-1}\right| T} \bar{\mu}=\bar{c}
$$

for all $t \geq 0$. From the definition of the saturation level $\bar{c}$ of $\sigma_{\bar{c}}$, it follows that for all $t \geq 0$, we have

$$
\left\{\begin{array}{l}
\dot{\tilde{z}}_{i}(t)=\tilde{z}_{i+1}(t), \quad i \in\{1, \ldots, k-1\} \\
\dot{\tilde{z}}_{k}(t)=\vartheta(\tilde{Z}(t))+\sum_{i=1}^{k} v_{i} \tilde{z}_{i}(t)
\end{array}\right.
$$

so the lemma follows from our choice of $\vartheta$ in Lemma 2.

We now combine the preceding lemmas to prove Theorem 1 . We begin by proving the first conclusion of the theorem, in which $\eta=0$. In this case, the closed loop system is

$$
\left\{\begin{aligned}
\dot{x}(t) & =\mathcal{F}(t, x(t), z(t), 0) \\
\dot{z}_{i}(t) & =z_{i+1}(t), i \in\{1, \ldots, k-1\} \\
\dot{z}_{k}(t) & =u\left(Z(t), Y_{t}, x_{t}\right)+\sum_{j=1}^{k} v_{j} z_{j}(t) \\
\dot{Y}(t) & =J_{2 k-1} Y(t)+\frac{e_{2 k-1}}{T} \omega(x(t)) .
\end{aligned}\right.
$$


Using the preceding lemma with $\mu_{0}(t)=\omega(x(t))$, we deduce that

$$
\lim _{t \rightarrow+\infty}\left|z_{i}(t)-\Omega_{i}(t)\right|=0
$$

for all $i=1$ to $k$, and $\tilde{z}_{i}=z_{i}-\Omega_{i}$ exponentially converges to 0 for all $i$.

Next notice that the $x$ subsystem of (52) can be written as

$$
\dot{x}(t)=\mathcal{F}(t, x(t), \Omega(t)+\tilde{z}(t), 0)
$$

when we choose the bounded function $\mu_{0}(t)=\omega(x(t))$. Hence, we can use Assumption 1 to conclude that $\lim _{t \rightarrow+\infty}|x(t)|=0$ and therefore that for all $i \in\{1,2, \ldots, k\}$, we have $\lim _{t \rightarrow+\infty} \Omega_{i}(t)=0$, since $\omega(0)=0$ and $\omega$ is continuous at 0 . It follows that

$$
\lim _{t \rightarrow+\infty} z(t)=\lim _{t \rightarrow+\infty}(z(t)-\Omega(t))+\lim _{t \rightarrow+\infty} \Omega(t)=0 .
$$

Since $\dot{Y}=J_{2 k-1} Y+\varepsilon$ is ISS with respect to $\varepsilon$, this proves the first conclusion of Theorem 1 .

It remains to prove the second conclusion of the theorem. To this end, first note that with the notation from our proof of the first conclusion of the theorem, the dynamics for $\tilde{z}$ are globally asymptotically stable to 0 , so the interconnection of the perturbed dynamics $\dot{x}(t)=\mathcal{F}(t, x(t), \Omega(t)+\tilde{z}(t), \eta(t))$ with $\mu_{0}^{\prime}(t)=\omega(x(t))$ and the $\tilde{z}$ dynamics will be ISS with respect to $\eta$, by standard small gain arguments. Then the structure of the function $\Omega$ implies that the $(x, z)=(x, \tilde{z}+\Omega)$ dynamics are ISS with respect to $\eta$. This completes the proof of our theorem.

\section{Extension to Systems with Measurement Delays}

Although [18] did not provide a bounded backstepping controller for the original system (1), it allowed cases where current values of the $x$ components of the state of the original system were not available for use in the control, leading to feedback controls in which $x(t)$ must be replaced by time delayed values $x(t-D)$ of $x$ for a constant delay $D>0$. In the same way, we can extend Theorem 1 above to allow cases where one must use time lagged values of $x$ instead of current ones. This is done by replacing $\omega(x(\ell))$ in the preceding analysis by $\omega(x(\ell-D))$ for constant values of the delay $D$, so instead of placing a converging-input-converging-state assumption on (9) in Assumption 1, we must replace (9) by the delayed version

$$
\dot{\xi}(t)=\mathcal{F}\left(t, \xi(t), \int_{t-T}^{t} \Lambda(\ell, t) \omega(\xi(\ell-D)) \mathrm{d} \ell+\delta(t), 0\right),
$$

and then the conclusions of the theorem remain true with $x(\ell)$ replaced by $x(\ell-D)$ in the feedback control. However, our sufficient conditions from Proposition 1 do not apply in cases such as (56) with measurement delays. This motivates the following analog of Proposition 1 that provides sufficient conditions for our delayed version of the converging-input-converging-state condition to hold, and which can therefore facilitate checking the requirements of our theorem when constant measurement delays $D$ are introduced in the $x$ measurements. In what follows, we use the same choices of $\Lambda_{a}(T)$ from (19) and $\Lambda_{+}(T)=\sup _{t>0}\left\{\left|\left(\Lambda_{1}(\ell, t), \ldots, \Lambda_{p}(\ell, t)\right)\right|\right.$ : $t-T \leq \ell \leq t\}$ as in Section 3, which are still independent of $t$, and which also do not depend on $D$.

Proposition 2. If Assumption 2 holds, and if the constants $T>0$ and $D>0$ are such that

$$
\begin{aligned}
& \mathcal{R}(T)<1 \text { and } r_{0} C \Lambda_{a}(T)\left(r_{2}+r_{3} \Lambda_{+}(T) r_{1}(D+T)\right)(D+T)<\frac{1-\mathcal{R}(T)}{8 \sqrt{2}}, \text { where } \\
& \mathcal{R}(T)=4\left(T \Lambda_{a}(T) r_{0} C\right)^{2}\left[2 r_{2}^{2}+\frac{5}{2}\left(r_{1} r_{3} T \Lambda_{+}(T)\right)^{2}\right]
\end{aligned}
$$

then the following is true: For each continuous function $\delta:[0,+\infty) \rightarrow \mathbb{R}^{p}$ that exponentially converges to zero, all solutions of (56) converge to 0 as $t \rightarrow+\infty$. If, in addition, the function $W$ from Assumption 2 is proper, then the system

$$
\dot{\xi}(t)=\mathcal{F}\left(t, \xi(t), \int_{t-T}^{t} \Lambda(\ell, t) \omega(\xi(\ell-D)) \mathrm{d} \ell+\delta(t), \eta(t)\right)
$$

is ISS with respect to $(\delta, \eta)$. 
Proof. We indicate the changes needed in the proof of Proposition 1. We let $c_{*}>0$ be the constant in curly braces in (28) as before, and where $\lambda$ is chosen as in the proof of Proposition 1. We may assume that $\lambda>1$ is close enough to 1 so that the requirements from (57) are still true if we replace $\mathcal{R}(T)$ by $\mathcal{R}(T)=4 \lambda\left(T \Lambda_{a}(T) r_{0} C\right)^{2}\left[2 r_{2}^{2}+2.5\left(r_{1} r_{3} T \Lambda_{+}(T)\right)^{2}\right]$ (by the strictness of the inequalities in (57)), and we make this replacement in the rest of the proof. Then, using our notation from the proof of Proposition 1, we have $c_{*}=0.5(1-\mathcal{R}(T))=0.5\left(1-4 T \mathcal{N}_{1} \lambda\right)$. In what follows, we use $\Lambda_{a}$ and $\Lambda_{+}$to mean $\Lambda_{a}(T)$ and $\Lambda_{+}(T)$, respectively, to keep our notation simple. Using the function $V$ from Assumption 2 and Young's Inequality, the additional term that must be added to the decay estimate on $V$ can be bounded above as follows:

$$
\begin{aligned}
& V_{x}(t, x(t)) g(t, x) \int_{t-T}^{t} \Lambda^{\mathrm{b}}(\ell, t)[\omega(x(\ell-D))-\omega(x(\ell))] \mathrm{d} \ell \leq C r_{0} \Lambda_{a} \sqrt{W(x(t))} \int_{t-T-D}^{t}|\dot{x}(s)| \mathrm{d} s \\
& \leq C r_{0} \Lambda_{a} \sqrt{W(x(t))} \int_{t-T-D}^{t}\left\{|f(s, x(s))|+|g(s, x(s))|\left[\Lambda_{+} \int_{s-T}^{s}|\omega(x(\ell-D))| \mathrm{d} \ell+|\eta(s)|\right]\right\} \mathrm{d} s \\
& \leq C r_{0} \Lambda_{a} \sqrt{W(x(t))} \int_{t-T-D}^{t}\left(r_{2} \sqrt{W(x(s))}+r_{3} \Lambda_{+} \int_{t-D-2 T}^{t}|\omega(x(\ell-D))| \mathrm{d} \ell+r_{3}|\eta(s)|\right) \mathrm{d} s \\
& \leq C r_{0} \Lambda_{a} \sqrt{W(x(t))}\left[\left(r_{2}+r_{3} \Lambda_{+} r_{1}(D+T)\right) \int_{t-2 D-2 T}^{t} \sqrt{W(x(\ell))} \mathrm{d} \ell+r_{3}(D+T)|\eta|_{[0, t]}\right] \\
& \leq \frac{c_{*}}{4} W(x(t))+\frac{1}{c_{*}}\left(r_{0} C \Lambda_{a}\left[\left(r_{2}+r_{3} \Lambda_{+} r_{1}(D+T)\right) \int_{t-2(D+T)}^{t} \sqrt{W(x(\ell))} \mathrm{d} \ell+r_{3}(D+T)|\eta|_{[0, t]}\right]\right)^{2} \\
& \leq \frac{c_{*}}{4} W(x(t))+\frac{4}{c_{*}}\left\{r_{0} C \Lambda_{a}\left(r_{2}+r_{3} \Lambda_{+} r_{1}(D+T)\right)\right\}^{2}(D+T) \int_{t-2 D-2 T}^{t} W(x(\ell)) \mathrm{d} \ell \\
& \quad+\frac{2}{c_{*}}\left(C r_{0} r_{3} \Lambda_{a}(D+T)\right)^{2}|\eta|_{[0, t]}^{2},
\end{aligned}
$$

where the last inequality also used Young's inequality, the relation $(a+b)^{2} \leq 2 a^{2}+2 b^{2}$ for suitable nonnegative values of $a$ and $b$, and then Jensen's inequality. Using the second inequality in (57) and choosing $\lambda>1$ close enough to 1 , it follows that we can find a constant $\lambda_{*}>1$ that is close enough to 1 and which is such that

$$
\frac{8 \lambda_{*}}{c_{*}}\left\{r_{0} C \Lambda_{a}(D+T)\left(r_{2}+r_{3} \Lambda_{+} r_{1}(D+T)\right)\right\}^{2}<\frac{c_{*}}{4}
$$

since $c_{*}=0.5(1-\mathcal{R}(T))$. Then reasoning analogously to the argument that produced (28) shows that the time derivative of

$$
\begin{aligned}
& V_{2}\left(t, x_{t}\right)= \\
& V_{1}\left(t, x_{t}\right)+\frac{4 \lambda_{*}}{c_{*}}\left\{r_{0} C \Lambda_{a}\left(r_{2}+r_{3} \Lambda_{+} r_{1}(D+T)\right)\right\}^{2}(D+T) \int_{t-2(D+T)}^{t} \int_{\ell}^{t} W(x(s)) \mathrm{d} s \mathrm{~d} \ell
\end{aligned}
$$

along all solutions of (58) admits positive constants $c_{* *}$ and $c_{* * *}$ such that $\dot{V}_{2} \leq-c_{* *} W(x(t))+c_{* * *}|(\delta, \eta)|_{[0, t]}^{2}$. If, in addition, $W$ is proper, then we can argue as in the proof of Proposition 1 to find a function $\gamma_{a} \in \mathcal{K}_{\infty}$ and a positive constant $k_{a}$ such that

$$
\dot{V}_{2} \leq-\gamma_{a}\left(V_{2}\left(t, x_{t}\right)\right)+k_{a}|(\delta, \eta)|_{[0, t]}^{2}
$$

(by using the bound (32) except with $T$ in (32) replaced by $D+T$ ). Then the rest of the proof is the same as in the last part of the proof of Proposition 1 except with $V_{1}$ replaced by $V_{2}$.

\section{Illustrations}

Our Lyapunov function based sufficient conditions are convenient for checking our assumptions from Theorem 1. We illustrate this point in this section, in two examples. In our first example, we apply our Lyapunov sufficient conditions directly. In our second example, our Lyapunov sufficient conditions do not apply directly, but we use a mixture of our Lyapunov and trajectory based methods to check our converging-input-convergingstate conditions. For simplicity, this section only considers cases where there are no measurement delays $D$, but we can apply the methods from the preceding section to cover measurement delays as well.

\subsection{First Illustration}

Consider the three-dimensional system

$$
\left\{\begin{aligned}
\dot{x}(t) & =\frac{|x(t)|}{1+|x(t)|}+z_{1}(t) \\
\dot{z}_{1}(t) & =z_{2}(t) \\
\dot{z}_{2}(t) & =u(t)
\end{aligned}\right.
$$


which is not amenable to classical backstepping, because the right side of $\dot{x}(t)$ in the dynamics is not differentiable. In terms of our notation from Section 3, we choose $k=2, n=1, q=1, p=1$, and

$$
\mathcal{F}\left(t, x, z_{1}\right)=\frac{|x|}{1+|x|}+z_{1} \text { and } \omega(x)=-\frac{1}{\Lambda_{*}(T)}\left(\frac{|x|}{1+|x|}+2 \frac{x}{1+|x|}\right),
$$

where

$$
\Lambda_{*}(T)=\int_{-T}^{0} \Lambda_{1}(\ell+t, t) \mathrm{d} \ell=\int_{-T}^{0} e^{q \ell} \ell^{k-1}(\ell+T)^{k-1} \mathrm{~d} \ell=2-T-e^{-T}(2+T) .
$$

We compute a constant $T>0$ such that Assumption 1 is satisfied. First note that since $p=1$, and since $\Lambda_{1}$ is nonpositive valued, we have $\Lambda_{a}(T)=-\Lambda_{*}(T)=\left|\Lambda_{*}(T)\right|$. Since (63) are globally Lipschitz functions and $\mathcal{F}$ is an affine function of $z_{1}$ and $\omega$ is bounded, it suffices to find constants $r_{i}$ for $i=0,1,2,3$ and functions $V$ and $W$ such that Assumption 2 is satisfied with

$$
f(t, x)=\frac{|x|}{1+|x|} \text { and } g(t, x)=1
$$

and then to choose $T$ such that our condition (20) holds.

To this end, we check that Assumption 2 is satisfied using the functions

$$
V(t, x)=\int_{0}^{x} \sigma_{1}(\ell) \mathrm{d} \ell \text { and } W(x)=\frac{2 \sigma_{1}(x) x}{1+|x|} .
$$

Since (65) give

$$
f(t, x)+g(t, x) \Lambda_{*}(T) \omega(x)=-\frac{2 x}{1+|x|}
$$

our conditions (18) on the $r_{i}$ 's from Assumption 2 for the preceding choices of $f, g, V$, and $W$ will be satisfied if

$$
\left|\sigma_{1}(x)\right| \leq r_{0} \sqrt{\frac{2 \sigma_{1}(x) x}{1+|x|}}, \frac{1}{\left|\Lambda_{*}(T)\right|} \frac{3|x|}{1+|x|} \leq r_{1} \sqrt{\frac{2 \sigma_{1}(x) x}{1+|x|}}, \frac{|x|}{1+|x|} \leq r_{2} \sqrt{\frac{2 \sigma_{1}(x) x}{1+|x|}} \text {, and } 1 \leq r_{3} .
$$

By separately considering points $x \in[-1,1]$ and points $x \notin[-1,1]$, it follows easily that Assumption 2 is satisfied with the choices

$$
C=\frac{3}{\left|\Lambda_{*}(T)\right|}, r_{0}=1, r_{1}=\frac{3}{\sqrt{2}\left|\Lambda_{*}(T)\right|}, r_{2}=1, \text { and } r_{3}=1 .
$$

Hence, our requirement (20) on $T>0$ from Proposition 1 holds if

$$
1>4(3 T)^{2}\left[2+\frac{5}{2}\left(\frac{3 T^{3}}{\sqrt{2}\left|2-T-e^{-T}(2+T)\right|}\right)^{2}\right]
$$

and we can use Mathematica [25] to check that the right side of (70) takes the value 0.912536 at $T=0.11$. Hence, Assumption 1 is satisfied with $T=0.11$, and then the desired controller is provided by Theorem 1 .

\subsection{Second Illustration}

We can sometimes apply Theorem 1 by checking Assumption 1 through a mixture of Lyapunov and direct trajectory analyses. For instance, consider the three dimensional system

$$
\left\{\begin{array}{l}
\dot{x}=x^{2}-x^{3}+z_{1} \\
\dot{z}_{1}=z_{2} \\
\dot{z}_{2}=u .
\end{array}\right.
$$

As noted in [8, pages 593-594], the system (71) is globally asymptotically stabilized to 0 by the control

$$
u(x, z)=-\frac{\partial V_{0}}{\partial z_{1}}\left(x, z_{1}\right)+\frac{\partial \phi}{\partial z_{1}}\left(x, z_{1}\right) z_{2}-z_{2}+\frac{\partial \phi}{\partial x}\left(x, z_{1}\right)\left(x^{2}-x^{3}+z_{1}\right)+\phi\left(x, z_{1}\right),
$$

where $V_{0}\left(x, z_{1}\right)=\frac{1}{2} x^{2}+\frac{1}{2}\left(z_{1}+x+x^{2}\right)^{2}$ and $\phi\left(x, z_{1}\right)=-2 x-(1+2 x)\left(x^{2}-x^{3}+z_{1}\right)-z_{1}-x^{2}$,

which is unbounded since it satisfies $\lim _{x \rightarrow \infty} u(x, 0)=-\infty$. Our work [18] provided the unbounded control

$$
u(t)=\frac{q^{2}}{\left(1-e^{-q \tau}\right)^{2}}\left\{J(x(t))-2 e^{-q \tau} J(x(t-\tau))+e^{-2 q \tau} J(x(t-2 \tau))\right\}-2 q z_{2}(t)-q^{2} z_{1}(t),
$$

where $J(x)=-\sin \left(\frac{\pi x}{2}\right) \mathbf{1}_{[-2,2]}(x)$ 
that rendered (71) globally asymptotically stable to 0 , where the indicator function $\mathbf{1}_{[-2,2]}$ is defined to be 1 on $[-2,2]$, and 0 on $\mathbb{R} \backslash[-2,2]$. Here we show how our new Theorem 1 provides a globally bounded globally asymptotically stabilizing controller for (71), using the choice of $\omega=J / \Lambda_{*}(T)$ with $J$ as defined in (72), and with $q=1, p=1$, and $k=2$ and with the artificial $T>0$ to be specified.

To verify Assumption 1 with the preceding choices, first note that for each continuous function $\delta: \mathbb{R} \rightarrow \mathbb{R}$ that exponentially converges to 0 and each initial state $x_{0} \in \mathbb{R}$, we can find a value $T_{*}\left(x_{0}, \delta\right) \in[0, \infty)$ such that the corresponding solution of

$$
\dot{x}(t)=x^{2}(t)-x^{3}(t)+\int_{t-T}^{t} \Lambda_{1}(\ell, t) \omega(x(\ell)) \mathrm{d} \ell+\delta(t)
$$

satisfies $x(t) \in[-0.8,3 / 2]$ for all $t \geq T_{*}\left(x_{0}, \delta\right)$. This can be done by noting that the integral in (73) is bounded by 1 , that $x^{2}-x^{3} \leq-1.125$ for all $x \geq 3 / 2$, and that $x^{2}-x^{3} \geq 1.152$ for all $x \leq-0.8$, so the right side terms $x^{2}(t)-x^{3}(t)$ in $(73)$ dominate the other right side terms, since we may assume that $t$ is large enough so that $|\delta(t)| \leq 0.12$. Hence, it suffices to check the inequalities (18) from Assumption 2 for all $x \in[-0.8,3 / 2]$, by only considering time values $t \geq T_{*}\left(x_{0}, \delta\right)$.

We now check the estimates from (18) for all $x \in[-0.8,3 / 2]$ using $V(x)=\frac{1}{2} x^{2}, W(x)=x^{2}, f(x)=x^{2}-x^{3}$, and $g(x)=1$. First note that simple calculations (e.g., using Mathematica [25]) give $x^{2}-x^{3}-\sin (\pi x / 2) \leq-x$ (resp., $\geq-x$ ) for all $x \in[0,3 / 2]$ (resp., $x \in[-0.8,0]$ ) which gives $\nabla V(x)(f(x)+\omega(x)) \leq-W(x),\left|x^{2}-x^{3}\right| \leq$ $1.44|x|$, and $|\sin (\pi x / 2)| \leq(\pi / 2)|x|$ when $x \in[-0.8,3 / 2]$, so we can choose $r_{0}=1, r_{1}=\pi /\left(2\left|\Lambda_{*}(T)\right|\right)$, $r_{2}=1.44, r_{3}=1$, and $C=\pi /\left(2\left|\Lambda_{*}(T)\right|\right)$. Hence, we can use our formula (64) for $\Lambda_{*}(T)$ and Mathematica to check that the sufficient condition (20) from Proposition 1 (for $\lim _{t \rightarrow \infty} x(t)=0$ to hold) is satisfied if

$$
1>(T \pi)^{2}\left(2(1.44)^{2}+\frac{5 \pi^{2}}{8}\left(\frac{T^{3}}{2-T-e^{-T}(2+T)}\right)^{2}\right)
$$

which is satisfied for all $T \in(0,0.0209]$. Therefore, we can satisfy our requirements with $T=0.0209$, and then the desired bounded control is provided by Theorem 1 .

\section{Conclusions}

We provided a new bounded backstepping technique for a large class of cascaded partially linear systems with arbitrarily large numbers of integrators, under a converging-input-converging-state assumption involving the nonlinear subsystems. For many cases where the nonlinear part of the system is control affine, we used Lyapunov functions to provide sufficient conditions for our converging-input-converging-state assumption to be satisfied. Although our controller involves a dynamic extension, it has an advantage that it provides bounded controllers for the original system, which would not have been possible under our assumptions if we had instead relied on previous results. We plan to combine our new methods with the time delay methods in [1] and [9] to also allow arbitrarily long measurement delays.

\section{References}

[1] N. Bekiaris-Liberis and M. Krstic. Nonlinear Control under Nonconstant Delays. Society for Industrial and Applied Mathematics, Philadelphia, PA, 2013.

[2] B. Bialy, J. Klotz, J. Curtis, and W. Dixon. Adaptive backstepping controller for a hypersonic airbreathing missile. In Proc. AIAA Guidance Navigation and Control Conference, Minneapolis, MN, Paper AIAA 2012-4468, 2012.

[3] W. Dixon, E. Zergeroglu, D. Dawson, and M. Hannan. Global adaptive partial state feedback tracking control of rigid-link flexible-joint robots. Robotica, 18:325-336, 2000.

[4] K. Dupree, C-H. Liang, G. Hu, and W. Dixon. Adaptive Lyapunov-based control of a robot and massspring system undergoing an impact collision. IEEE Transactions on Systems, Man, and Cybernetics Part B: Cybernetics, 38(4):1050-1061, 2008. 
[5] R. Freeman and L. Praly. Integrator backstepping for bounded controls and control rates. IEEE Transactions on Automatic Control, 43(2):258-262, 1998.

[6] A. Isidori. Nonlinear Control Systems, Third Edition. Springer. New York, 1995.

[7] Z-P. Jiang and H. Nijmeijer. A recursive technique for tracking control of nonholonomic systems in chained form. IEEE Transactions on Automatic Control, 44(2):265-279, 1999.

[8] H. Khalil. Nonlinear Systems, Third Edition. Prentice Hall, Upper Saddle River, NJ, 2002.

[9] M. Krstic. Delay Compensation for Nonlinear, Adaptive, and PDE Systems. Birkhauser, Boston, 2009.

[10] M. Krstic, I. Kanellakopoulos, and P. Kokotovic. Nonlinear and Adaptive Control Design. John Wiley and Sons, New York, NY, 1995.

[11] M. Malisoff and F. Mazenc. Constructions of Strict Lyapunov Functions. Springer-Verlag, London, 2009.

[12] F. Mazenc and S. Bowong. Backstepping with bounded feedbacks for time varying systems. SIAM Journal on Control and Optimization, 43(3):856-871, 2004.

[13] F. Mazenc and A. Iggidr. Backstepping with bounded feedbacks. Systems and Control Letters, 51(34):235-245, 2004.

[14] F. Mazenc and M. Malisoff. Bounded backstepping approach under input delays. In Proc. European Control Conference, pages 2056-2061, Linz, Austria, 2015.

[15] F. Mazenc and M. Malisoff. New control design for bounded backstepping under input delays. Automatica, 66:48-55, 2016.

[16] F. Mazenc, M. Malisoff, and L. Burlion. Bounded backstepping through a dynamic extension with delay. In Proc. IEEE Conference on Decision and Control, 2017, to appear.

[17] F. Mazenc, M. Malisoff, and Z. Lin. Further results on input-to-state stability for nonlinear systems with delayed feedbacks. Automatica, 44(9):2415-2421, 2008.

[18] F. Mazenc, M. Malisoff, and J. Weston. New bounded backstepping control designs for time-varying systems under converging input converging state conditions. In Proc. IEEE Conference on Decision and Control, pages 3167-3171, Las Vegas, NV, USA, 2016.

[19] F. Mazenc, S-I. Niculescu, and M. Bekaik. Backstepping for nonlinear systems with delay in the input revisited. SIAM Journal on Control and Optimization, 49(6):2263-2278, 2011.

[20] E. Sontag. Mathematical Control Theory: Deterministic Finite-Dimensional Systems. Springer, New York, NY, 1998.

[21] H. Sussmann, E. Sontag, and Y. Yang. A general result on the stabilization of linear systems using bounded controls. IEEE Transactions on Automatic Control, 39(12):2411-2425, 1994.

[22] J. Tsinias. Input to state stability properties of nonlinear systems and applications to bounded feedback stabilization using saturation. ESAIM Control, Optimization, and Calculus of Variations, 2:57-87, 1997.

[23] J. Tsinias. Backstepping design for time-varying systems and application to partial-static feedback and asymptotic tracking. Systems and Control Letters, 39:219-227, 2000.

[24] J. Tsinias and I. Karafyllis. ISS property for time-varying systems and application to partial-static feedback stabilization and asymptotic tracking. IEEE Transactions on Automatic Control, 44(11):2179 2184, 1999.

[25] Wolfram Mathematica. The world's definitive system for modern technical computing, 2015. Wolfram Research, http://www.wolfram.com/mathematica/, Accessed August 17, 2017. 